\title{
LARINGOTRAQUEOSCOPIA FLEXÍVEL NA DECANULAÇÃO DE PACIENTES TRAQUEOSTOMIZADOS: OTIMIZAÇÃO DE SEGURANÇA PARA O PACIENTE
}

Mauricio Medeiros Lemos

Universidade Estadual de Maringá - UEM. Programa de Ciências da Saúde, Brasil.

\section{César Orlando Peralta Bandeira}

Docente associado da disciplina de cirurgia torácica da Universidade Estadual de Maringá - UEM, Maringá/ PR, Brasil

\section{Vlaudimir Dias Marques}

Docente associado da Universidade Estadual de Maringá - UEM, Maringá/PR, Brasil.

Jorge Juarez Vieira Teixeira

Docente associada da Universidade Estadual de Maringá, UEM, Maringá/PR, Brasil.

\section{Maria Dalva Barros de Carvalho}

Docente associado $\mathrm{C}$ da Universidade Estadual de Maringá - UEM, Brasil.
Autor correspondente:

Mauricio Medeiros Lemos

maulemostb@hotmail.com
RESUMO: Avaliar a decanulação utilizando a laringotraqueoscopia flexível visando o reconhecimento das lesões laringotraqueais não diagnosticadas quando utilizados somente critérios clínicos. Participaram do estudo 100 pacientes, com idade entre 18 e 80 anos, traqueostomizados, com indicação de decanulação que foram submetidos à oclusão da cânula por 03 minutos para avaliar fonação e ventilação seguido da realização do exame. Foi comparada a indicação de decanulação por critérios clínicos com o diagnóstico de lesões laringotraqueais reconhecidas após o término da laringotraqueoscopia flexível. Os critérios de Myer-Cotton foram utilizados como referencial para classificação de doença laringotraqueal. Resultados: 62 (62\%) pacientes apresentaram critérios clínicos para decanulação, porém, com doença laringotraqueal identificada pela laringotraqueoscopia flexível. Dentre estes, oito (8\%) considerados aptos à decanulação baseados nos critérios clínicos apresentaram contraindicação à retirada da cânula. Apenas 26 (26\%) pacientes não apresentaram doença laringotraqueal; 11 (11\%) não foram decanulados por não preencherem critérios clínicos e endoscópicos. A laringotraqueoscopia flexível reconheceu doença laringotraqueal nos pacientes que preenchiam critérios clínicos para retirada da cânula traqueal. Este exame como rotina na avaliação de pacientes preenchendo critérios de decanulação mostrou-se útil e seguro.

PALAVRAS-CHAVE: Decanulação; Doenças traqueais; Medidas de segurança; Obstrução de via aérea; Traqueostomia.

\section{FLEXIBLE LARINGOTRACHEOSCOPY IN DE- CANNULATION OF TRACHEOSTOMIZED PATIENTS: SAFETY IMPROVEMENTS FOR THE PATIENT}

ABSTRACT: To evaluate de-cannulation by flexible laringeotracheoscopy for laringeotracheal lesions which are not diagnosed by clinical criteria. One hundred tracheostomized patients, aged between 18 and 80 years old, with indication of de-cannulation submitted to occlusion of the cannula during three minutes to evaluate fonation and ventilation, followed by tests. De-cannulation by clinical criteria was compared with diagnosis of larigeotracheal lesions after the end of flexible laringeotracheoscopy. Myer-Cotton criteria were employed, a referential for the classification of laringeotracheal diseases. Sixty-two (62\%) patients had clinical criteria for de-cannulation but with laringeotracheal disease identified by flexible largineotracheoscopy. Eight (8\%) could be de-cannulated, based on clinical criteria which counterindicated the removal of the cannula. Only twenty-six (26\%) did not present a laringeotracheal disease. Eleven (11\%) were not de-cannulated since they did not comply with clinical and endoscopy criteria. Flexible laringeotracheoscopy acknowledged the laringeotracheal disease in patients who complied with clinical criteria for the removal of tracheal cannula. The test, as a routine in patients' evaluation complying with de-cannulation criteria, was useful and safe. 
KEY WORDS: De-cannulation; tracheal diseases; safety measures; obstruction of aerial pathways; Tracheostomy.

\section{INTRODUÇÃO}

Nos Estados Unidos, a cada ano, mais de 950.000 pacientes passam por ventilação mecânica devido à insuficiência respiratória aguda, muitas vezes por um período de dias semanas ou meses. A literatura descreve que há uma grande parcela, atualmente em torno de um quarto destes pacientes que necessitam de ventilação mecânica por mais de 48 horas necessitaram de traqueostomia para ventilação mecânica prolongada. ${ }^{1}$

A traqueostomia é um dos procedimentos mais comuns na unidade de terapia intensiva. Cerca de $10 \%$ dos pacientes que necessitam de ventilação mecânica, no período entre 7 a 14 dias ao final são traqueostomizados para manutenção de ventilação mecânica prolongada ou suporte de vias aéreas. ${ }^{1,2,3}$

Sabe-se que a permanência prolongada do tubo orotraqueal é fator causador de complicações anatomofisiológicas laringotraqueais e potencial gerador de sequelas graves em via aérea. ${ }^{4}$ As alterações laríngeas agudas são diagnósticas em até $94 \%$ dos pacientes, desde um processo inflamatório traqueal à estenose complexa. $\mathrm{O}$ mecanismo fisiopatológico conhecido dá-se secundário a isquemia promovida a membrana traqueal pelas altas pressões do cuff. A substituição do tubo pela traqueostomia reduz o índice dessas complicações. ${ }^{5,6}$ Outra indicação da traqueostomia é a obtenção de via aérea segura nos pacientes com risco de obstrução das vias aéreas superiores, sobretudo por processos infecciosos cervicais profundos, tumores, corpo estranho, angioedema, disfunção de pregas vocais e edema glótico após intubação orotraqueal. ${ }^{2,7,8}$

A cânula de traqueostomia, no entanto, apresenta-se como corpo estranho e pode causar complicações tais como broncorreia excessiva ou tosse; alteração do mecanismo fisiológico de proteção contra a broncoaspiração; diminuição da eficácia da tosse ; infecção; sangramento; dificuldade de vocalização, o que, além de provocar alterações laríngeas como hipotonia, contribui para o isolamento social do paciente. ${ }^{4,9,10}$
Em pacientes que utilizam cânula de traqueostomia, a alteração do processo fisiológico impedindo que o ar não passe pela cavidade nasofaríngea entrando diretamente a árvore traqueobrônquica prejudica todo sistema de umidificação do ar. Secundário ao déficit de umidificação, o epitélio traqueal é envolvido em um progressivo processo inflamatório resultando em metaplasia escamosa e, consequentemente, comprometimento da função ciliar aumentando o risco de infecção respiratória. ${ }^{4}$

Visando diminuir a incidência de complicações deve-se priorizar a realização da decanulação precocemente, desde que o paciente apresente condições adequadas. ${ }^{5,11-14}$

Foi focada a segurança baseada na realização da laringotraqueoscopia flexível visando melhor compreensão dos mecanismos fisiológicos da ventilação durante o processo de decanulação.

Atualmente há limitações da literatura em definir estrutura protocolar baseada em evidências indicando os critérios e o melhor momento para a decanulação. Os critérios clínicos empíricos de fonação e ventilação são amplamente utilizados. ${ }^{15,17}$

Até aonde se sabe não há estudos evidenciando a segurança da decanulação por meio da laringotraqueoscopia flexível. No sentido de identificar e avaliar as potenciais complicações, a laringotraqueoscopia flexível está indicada.

Embora pouco utilizada e sem protocolos específicos essa tecnologia auxilia na decisão sobre o momento da decanulação além de identificar as lesões laringotraqueais que necessitam de seguimento.

O objetivo deste estudo foi avaliar a utilização da laringotraqueoscopia flexível na decanulação de pacientes que já tinham indicação de retirada de cânula traqueal.

\section{METODOLOGIA}

Estudo clínico retrospectivo observacional, com pacientes traqueostomizados em processo de decanulação, realizados em uma clínica na cidade de Maringá-PR.

Os dados foram coletados de março de 2013 a janeiro de 2015. Todas as traqueostomias foram realizadas pela equipe de cirurgia torácica de acordo com a técnica 
padronizada na instituição: incisão transversal da pele, dissecção dos planos pré-traqueais e traqueotomia transversa no nível do segundo anel traqueal na parede anterior da traqueia. O estudo recebeu aprovação do Comitê de Ética em Envolvimento Humano (COPEP) do Centro Universitário Ingá (UNINGÁ), sob registro no 2.168.702.

Os critérios de inclusão foram a estabilidade clínica, em ventilação espontânea há pelo menos 48 horas, a ausência de infecção quando indicada a decanulação, a tosse e deglutição eficazes, a escala de coma de Glasgow maior que oito e em uso da cânula metálica de traqueostomia número 4 standart com diâmetro interno de $7,5 \mathrm{~mm}$ e externo de $10,4 \mathrm{~mm}$ e comprimento de $7 \mathrm{~cm}$. Foram excluídos os pacientes menores de 18 anos e as traqueostomias realizadas por outros serviços.

Os pacientes em vias de decanulação foram avaliados por médicos do serviço, que os assistiam na chegada à clínica. Os médicos foram responsáveis pela avaliação clínica e condição neurológica conforme escala de Glasgow. A tosse eficaz foi avaliada subjetivamente. Sua eficácia foi determinada pela possibilidade do paciente expelir secreção de vias aéreas pelo traqueostoma ou orofaringe.

Os pacientes foram submetidos à laringotraqueoscopia flexível pelos médicos do serviço. O exame foi realizado na sala de endoscopia respiratória da clínica, sendo utilizado o broncoscópio flexível Olympus, modelo BF-PE2, óptico, com diâmetro externo do canal de trabalho de 4,9 mm. Utilizou-se o protocolo de realização de exames do serviço: $8 \mathrm{~mL}$ de lidocaína gel na cavidade nasal; introdução do endoscópio pela cavidade nasal avaliando-se anatomia e fisiologia laríngea, mobilidade e morfologia das pregas vocais e região subglótica; identificação da cânula de traqueostomia, que foi retirada para melhor avaliação da traqueia. Após término do exame, a cânula de traqueostomia era retirada se o paciente apresentasse condições para decanulação.

Após o procedimento, os pacientes eram avaliados quanto à frequência respiratória, ausculta pulmonar, expansibilidade torácica e estabilidade hemodinâmica.

Definiu-se como condições ideais para decanulação: obstrução da luz traqueal cervical ou mediastinal menor que $50 \%$ baseado na classificação de Myer-Cotton. Nos pacientes não aptos à decanulação, observados pela laringotraqueoscopia flexível, a cânula foi reintroduzida e os pacientes orientados a manter seguimento no serviço.

Quadro 1. Classificação de Myer-Cotton para estenoses subglóticas

\begin{tabular}{|c|c|}
\hline Classificação & Obstrução lúmen (\%) \\
\hline Grau I & $<50 \%$ de obstrução do lúmen laríngeo \\
\hline Grau II & 50 a $70 \%$ de obstrução do lúmen laríngeo \\
\hline Grau III & 71 a $99 \%$ de obstrução do lúmen laríngeo \\
\hline Grau IV & Obstrução completa do lúmen laríngeo \\
\hline
\end{tabular}

Os dados foram organizados e tabulados no programa Excel $^{\circledR} 2007$ e análise estatística foi realizada no programa Stata ${ }^{\circledR} 9.1$ (Stata Corporation, College Station, TX, USA), de acordo preditores e desfecho de interesse. O teste de qui-quadrado, o Odds Ratio (OR) e respectivos intervalos de confiança (IC) foram calculados para cada variável. Um $\mathrm{p}<0,05$ foi considerado como estatisticamente significante.

\section{RESULTADOS}

Neste estudo foram avaliados 100 pacientes, sendo 55 do sexo feminino, média de idade de 44 anos \pm 18,5 com variação de 18 a 80 anos de idade. Acima de $50 \%$ dos pacientes apresentaram fonação e ventilação adequadas, toleraram a oclusão da cânula no início do exame por 3 minutos, apresentaram doença laringotraqueal grau I de Myer-Cotton e foram decanulados. Oito (8\%) pacientes apresentaram fonação e ventilação adequadas, toleraram a oclusão da cânula no início do exame por 3 minutos, apresentaram doença laringotraqueal grau II de Myer-Cotton e não foram decanulados. Vinte e sete pacientes (27\%) não apresentaram nenhuma doença laringotraqueal e foram decanulados conforme o protocolo estabelecido no estudo. Em número menor, $8 \%$, dos casos avaliados não apresentaram fonação, não toleraram a oclusão da cânula por 3 minutos e apresentaram doença laringotraqueal não foram decanulados. 


\section{0 pacientes - Indicação de Decanulação}
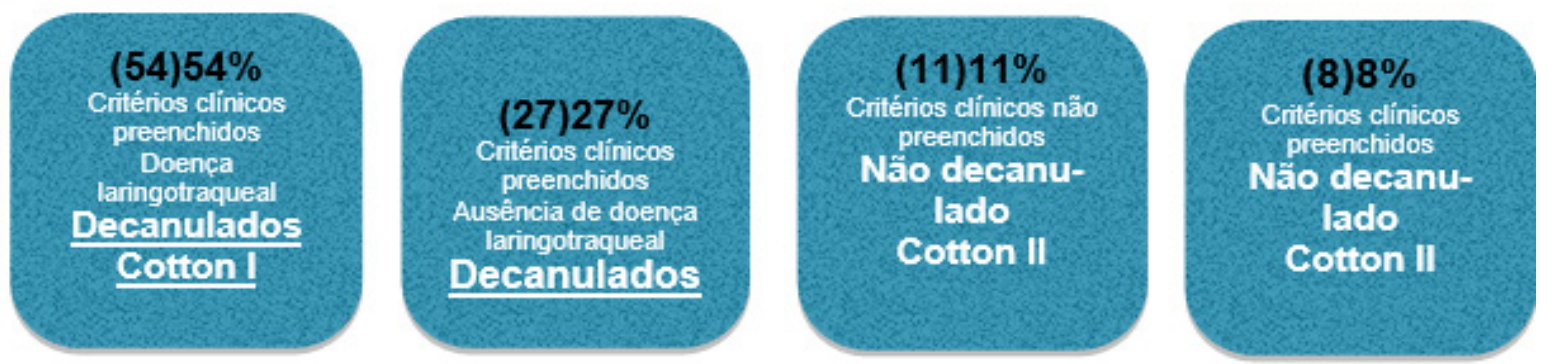

Figura 1. Demonstrativo de resultados em indicação de decanulação.

Os pacientes com fonação apresentaram 27 vezes mais chance para decanulação comparado aos casos sem fonação (Tabela 1).

Os pacientes com oclusão da cânula apresentaram 41 vezes mais chance para decanulação comparado aos casos sem oclusão de cânula (Tabela 1).

Tabela 1. Associação entre as variáveis preditoras e a decanulação - Maringá - PR 2016

\begin{tabular}{lllll}
\hline Variáveis & \multicolumn{2}{l}{ Decanulação } & \multicolumn{2}{l}{ Análise univariada } \\
\cline { 2 - 4 } preditoras & Sim & Não & $\mathrm{OR}^{*}(95 \% \mathrm{CI})$ & p valor \\
\hline & $\mathrm{n}=81(\%)$ & $\mathrm{n}=19(\%)$ & \\
\hline
\end{tabular}

\section{Fonação}

Sim $\quad 89(91,1) \quad 8(72,7) \quad 27,0(5,9-122,5) \quad<0,001$

Não $\quad 11(8,9) \quad 11(27,3) \quad 1$

Oclusão da cânula

\begin{tabular}{lllll}
\hline Sim & $89(91,1)$ & $8(20,0)$ & $41,0(7,4-226,9)$ & $<0,001$ \\
\hline Não & $11(8,9)$ & $11(80,0)$ & 1 & \\
\hline *OR (Odds Ratio)
\end{tabular}

Não houve associação estatística significante entre as variáveis preditoras, fonação e oclusão da cânula com a variável desfecho, doença laringotraqueal (Tabela 2).
Tabela 2. Associação entre as variáveis preditoras e a doença laringotraqueal - Maringá - PR 2016

\begin{tabular}{|c|c|c|c|c|}
\hline \multirow{2}{*}{$\begin{array}{l}\text { Variáaveis pre- } \\
\text { ditoras }\end{array}$} & \multicolumn{2}{|c|}{ Doença laringotraqueal } & \multicolumn{2}{|c|}{ Análise univariada } \\
\hline & Sim & Não & $\mathrm{OR}^{*}(95 \% \mathrm{CI})$ & $\begin{array}{c}\mathrm{p} \\
\text { valor }\end{array}$ \\
\hline & $n=73(\%)$ & $27(\%)$ & & \\
\hline \multicolumn{5}{|l|}{ Fonação } \\
\hline Sim & $62(86,1)$ & $27(96,4)$ & & 0,149 \\
\hline Não & $11(13,9)$ & $0(3,6)$ & $0,2(0,0-1,9)$ & \\
\hline \multicolumn{5}{|c|}{ Oclusão da cânula } \\
\hline Sim & $62(86,1)$ & $27(100,0)$ & $0,2(0,0-1,5)$ & 0,141 \\
\hline Não & $11(13,9)$ & $0(0,0)$ & 1 & \\
\hline
\end{tabular}

Tabela 3. Associação entre a decanulação e doença laringotraqueal - Maringá - PR 2016

\begin{tabular}{lllll}
\hline Variável & \multicolumn{2}{l}{ Doença laringotraqueal } & \multicolumn{2}{l}{ Análise univariada } \\
\cline { 2 - 5 } & Sim & Não & $\mathrm{OR}^{*}(95 \% \mathrm{CI})$ & $\mathrm{p}$ valor \\
\hline \multicolumn{4}{l}{$\mathrm{n}=73(\%)$} & $27(\%)$ \\
\hline Decanulação & & & & \\
\hline Sim & $81(77,8)$ & $27(100,0)$ & $0,1(0,0-0,8)$ & 0,019 \\
\hline Não & $19(22,2)$ & $0(0,0)$ & 1 & \\
\hline
\end{tabular}

*OR (Odds Ratio)

\section{DISCUSSÃO}

Objetivou-se a realização da laringotraqueoscopia flexível na avaliação da decanulação pela sensibilidade do método para diagnóstico de lesões anatômicas e reconhecimento de necessidade de seguimento especializado nos pacientes traqueostomizados. Este, até onde se 
sabe, é um dos primeiros estudos a avaliar a decanulação baseada no exame de laringotraqueoscopia flexível. Sachdev considera o exame extremamente seguro e com índices muito baixos de complicações. ${ }^{12}$

Foi considerado contraindicação à decanulação o diagnóstico de lesões que comprometiam $50 \%$ ou mais do diâmetro traqueal baseado na classificação de Myer-Cotton ${ }^{6}$. A partir deste grau de obstrução podem ocorrer alterações significativas nos testes de função pulmonar e repercussões clínicas. ${ }^{18}$ Entretanto, Grillo ${ }^{14}$, descreve que são significativas as obstruções que comprometem $20 \%$ do diâmetro traqueal.

Os resultados deste estudo fundamentaram a retirada da cânula utilizando o critério de $50 \%$ ou menos de obstrução baseado na utilização do exame endoscópico mostrando uma nova perspectiva para pacientes com doença traqueal.

O referencial da cânula metálica número 4 foi utilizado tendo em vista que seu diâmetro externo possui $10 \mathrm{~mm}$ e quando ocluída representa $50 \%$ de obstrução da luz traqueal. Em um indivíduo adulto, o diâmetro traqueal é de $20 \mathrm{~mm}$ nas mulheres e $23 \mathrm{~mm}$ nos homens. ${ }^{12,19}$

Entretanto, em $8 \%$ dos casos, que apresentaram critérios clínicos definidos, foram contraindicados para a decanulação baseado no critério endoscópico de Myer-Coton (Grau II). Rodrigues e Nunes ${ }^{11}$ utilizaram a broncoscopia flexível como um dos critérios de decanulação evidenciando achado de $20 \%$ de lesões laringotraqueais que poderiam determinar falha no processo de decanulação.

Kutsukutsa et al. ${ }^{16}$ sugeriram que a fundamentação de decanulação baseada em critérios clínicos exclusivos pode ser realizada, porém cada caso deveria ser avaliado individualmente, o que contraria os resultados deste trabalho. Essa discordância pode ser creditada à metodologia incluindo quadro clínico, doença de base, critérios de inclusão, tipo de cânula e diferença na descrição das lesões laringotraqueais em comparação com o presente estudo. ${ }^{16} \mathrm{~A}$ evidência de doença traqueal reconhecida pelo exame e a realização de decanulação fundamenta o diferencial de segurança aplicado ao paciente utilizando laringotraqueoscopia comparado com a aplicação de critérios somente clínicos. A laringotraqueoscopia beneficiou 62 (62\%) dos pacientes, uma vez que 54 deles possuíam doença laringotraqueal mas se enquadraram no critério endoscópico de decanulação. Em 8(8\%) foi reconhecida doença laringotraqueal que contraindicava a decanulação, mesmo cumprindo os critérios clínicos.

Dos indivíduos avaliados, 26 (26\%) apresentaram fonação à oclusão da cânula, toleraram a oclusão no início do exame por 3 minutos e não apresentaram qualquer tipo de doença laringotraqueal, sendo decanulados. Um estudo multicêntrico avaliou a opinião de 225 médicos referente às práticas contemporâneas de decanulação onde foi considerado o nível de consciência, tosse eficaz, secreções e oxigenação como fatores determinantes para recomendar a decanulação..$^{17,20} \mathrm{O}$ presente estudo evidenciou que apenas as evidências clínicas não são suficientemente seguras para definir a decisão de decanulação do paciente. $\mathrm{O}$ desenvolvimento de diretrizes baseadas em evidências para a traqueostomia irá facilitar a gestão segura e eficácia dos pacientes. ${ }^{17}$

Já para os 11\% dos casos avaliados com indicação para decanulação que não apresentaram fonação, não toleraram a oclusão da cânula por 3 minutos e apresentaram doença laringotraqueal não foram decanulados.

Este estudo evidenciou os benefícios de utilização da laringotraqueoscopia flexível como ferramenta de segurança aos pacientes. Rodrigues e Nunes ${ }^{11}$ consideraram em seu estudo que a broncoscopia beneficiou os pacientes como critério de decanulação, entretanto foi impossível identificar os fatores que tornam imprescindíveis a utilização do exame para decanular o paciente. Os resultados do presente estudo ao contrário, buscou definir a laringotraqueoscopia flexível como procedimento de rotina para a segurança do paciente em protocolos de decanulação.

Na pesquisa utilizou-se o protocolo do serviço composto de critérios clínicos e endoscópicos, que não são conhecidos entre a maioria dos investigadores pelo baixo número de estudos na área. São necessários mais estudos prospectivos com uma amostra maior, com intuito de determinar a importância da laringotraqueoscopia flexível na avaliação da decanulação com segurança. Estudos que abordam técnicas de avaliação que descrevam de maneira objetiva e padronizada as lesões laringotraqueais, podendo inserir como rotina os critérios endoscópicos de decanulação como os efetivados no presente estudo.

Pode-se afirmar, de acordo com os resultados 
encontrados nesta pesquisa, que a laringotraqueoscopia flexível evidenciou um número elevado de lesões laringotraqueais em pacientes que apresentavam critérios clínicos para a retirada da cânula. Identificou também em oito pacientes, que atendiam os critérios clínicos para decanulação, lesões laringotraqueais incompatíveis com a retirada segura da cânula.

\section{CONCLUSÃO}

O seguimento especializado e a segurança do paciente foram otimizadas inserindo a laringotraqueoscopia flexível no protocolo de decanulação de pacientes traqueostomizados. O reconhecimento de doença traqueal em pacientes clinicamente liberados para retirada da cânula ratifica a importância da necessidade do exame para os doentes.

\section{REFERÊNCIAS}

1. Pham T, Brochard LJ, Slutsky AS. Mechanical Ventilation: State of the art. Mayo Clin Proc. 2017; 92(9):1382-1400.

2. Schroder JB, Marian T, Muhle P, Claus I, Thomas C, Ruck T, et al. Intubation, tracheostomy, and decannulation in patients with Guillain-Barré-syndrome-does dysphagia matter? Muscle Nerve. 2019; 59(2):194200 .

3. Singh RK, Saran S, Baronia AK. The practice of tracheostomy decannulation - A systematic review. J Intensive Care. 2017; 5: 38.

4. Enrichi C, Battel L, Zanetti C, Koch I, Ventura L, Palmer K, et al. Clinical criteria for tracheostomy decannulation in subjects with acquired brain injury. Respir Care. 2017; 62(10):1255-1263.

5. Higashi T, Eguchi H, Wakayama Y, Sumi M, Saito T, Inaba Y. Analysis of the risk factors for tracheostomy and decannulation after traumatic cervical spinal cord injury in an aging population. Spinal Cord. 2019; 10. doi: 10.1038/s41393-019-0289-x.

6. Cotton, R T. Pediatric laryngotracheal stenosis. J Pediatr Surg. 1984; 1 9(6):699-704.

7. Southcott AM, Holdsworth C, Malcolm L, Muruganandan S, Skinner E. Evaluation of the implemen- tation of a Tracheostomy Review Services (TRS): an observational cohort study. J Interprof Care. 2019; 23:1 - 9 .

8. Budweiser S, Baur T, Jorres RA, Kollert F, Pfeifer M, Heinemann F. Predictors of successful decannulation using tracheostomy retainer in patients with prolonged weaning and persisting respiratory failure. Respiration. 2012; 84:469-476.

9. Mah JW, Staff II, Fisher SR, Butler KL. Improving decannulation and dwallowing function: A comprehensive, multidisciplinary approach to post-tracheostomy care. Respir Care. 2017; 62(2):137-143.

10. Nakarada-Kordic I, Patterson N, Wrapson J, Reay SD. A Systematic Review of Patient and Caregiver Experiences with a Tracheostomy. Patient. 2018; 11(2):175191.

11. Rodrigues LB; Nunes TA. Importância da broncoscopia flexível na decanulação de pacientes traqueostomizados. Rev. Col. Bras. Cir. 2015; 42(2): 075-080.

12. Sachdev A, Ghimiri A, Gupta N, Gupta D. Pre - decannulation flexible bronchoscopy in tracheostomized children. Pediatr Surg Int. 2017; 33(11):1195-1200.

13. Cohen O, Tzelnick S, Lahav Y, Stavi D, Shoffel-Havakuk $\mathrm{H}$, Hain $\mathrm{M}$, et al. Feasibility of a single-stage tracheostomy decannulation protocol with endoscopy in adult patients. Laryngoscope. 2016; 126(9):205762 .

14. Grillo HC. Surgery of the trachea and bronchi. Hamilton: BC Decker; 2004.

15. Welton C, Morrison M, Catalig M, Chris J, Pataki J, Can an interprofessional tracheostomy team improve weaning to decannulation times? A quality improvement evaluation. Can J Respir Ther. 2016; Winter; 52(1): 7-11.

16. Kutsukutsa J, Mashamba -Thompson TP, Saman Y. Tracheostomy decannulation methods and procedures in adults: A systematic scoping review protocol. Syst Rev. 2017; 4; 6 (1):239.

17. Santus P, Gramegna A, Radovanovic D, Racanelli R, Valenti V, Rabbiosi D, et al. A systematic review on tracheostomy decannulation: A proposal of a quantitative semiquantitative clinical score. Med. 2014; 15; 14:201. 
18. Brunet J, Dufour-Trivini M, Sauneuf B, Terzi N. Gestion de la décanulation: quelle prise en charge pour le patient trachéotomisé ? Management of decanulation in the tracheostomized patient. Réanimation, 2015.

19. Otorhinolaryngol. Tracheal decannulation protocol in patients affected by traumatic brain injury. In: Arch Otorhinolaryngol. 2014; 18(2):108-14.

20. Seligman KL, Liming BJ, Smith RJH. Pediatric Tracheostomy Decannulation: 11-Year Experience. Otolaryngol Head Neck Surg. 2019; Apr 16:194599819842164. doi: 10.1177/0194599819842164.

Recebido em: 18/02/2019

Aceito em: 06/06/2019 